\title{
A room-based diagnostic imaging system for measurement of patient setup
}

\author{
James E. Schewe, ${ }^{\text {a) }}$ Kwok L. Lam, James M. Balter, and Randall K. Ten Haken \\ Department of Radiation Oncology, University of Michigan, Ann Arbor, Michigan 48109-0010
}

(Received 10 December 1997; accepted for publication 17 September 1998)

\begin{abstract}
A room-based diagnostic x-ray imaging system for routine measurement of radiotherapy patient orientation has been developed. The system consists of a pair of room-mounted x-ray tubes and a portable imager consisting of an orthogonal pair of phosphor screens, a mirror/lens system, a CCD camera, and computer software for comparing images of the patient to reference images. Orthogonal pairs of images can be acquired quickly and with relatively little exposure, allowing correction of patient setup on a daily basis. This could limit patient setup error to the uncertainty in the measurement and repositioning processes, a potentially significant improvement over the present standard. (C) 1998 American Association of Physicists in Medicine. [S0094-2405(98)00612-9]
\end{abstract}

Key words: patient setup, conformal radiotherapy, diagnostic x-rays, portal imaging

Conformal radiation therapy treatments require accurate knowledge of patient orientation. Accounting for variations in patient setup requires the addition of margins to the target which can lead to treating a significant volume of normal tissue. The present standard of practice is to align patients daily with lasers and skin marks, and check the setup with port films about once a week. An earlier study in our clinic ${ }^{1}$ showed that this practice results in one standard deviation variations of 5-7 $\mathrm{mm}$ for each of three translations and 2.53.0 degrees for each of two rotations for patients treated to the pelvis, abdomen, and chest. These results are consistent with studies at other institutions. ${ }^{2-6}$ Monitoring and adjusting patient setup on a daily basis could reduce these variations to a level consistent with the uncertainty inherent in the measurement and repositioning process, ${ }^{7}$ but is only feasible with a system that provides accurate information quickly and with a small dose to the patient.

Diagnostic energy $\mathrm{x}$-rays provide superior image quality with a relatively low dose at treatment depth. A coordinate system that is fixed to the room, similar to wall-mounted alignment lasers, provides accuracy and stability, and allows for the use of complex, noncoplanar treatment fields without introducing additional collision avoidance constraints. Diagnostic imaging systems mounted on the treatment gantry have been used at other institutions previously. ${ }^{8-10}$

The patient orientation is found by comparing the acquired images to reference images using a graphical interface based on curve matching of bony anatomy. ${ }^{11}$ This yields a two-dimensional transformation (two translations and one inplane rotation) between each image and its corresponding reference image. An orthogonal pair of planar transformations gives an approximate three-dimensional representation of the patient orientation. The random uncertainty (one standard deviation) in alignment of bony anatomy is approximately 1.2 degrees rotation and $1.2 \mathrm{~mm}$ for each of the two translations. ${ }^{1}$ The imaging system is designed to be used with a computer-controlled radiotherapy system ${ }^{12}$ (CCRS) and a treatment couch incorporating tilt and roll ${ }^{13}$ to adjust patient orientation.

The system is shown schematically in Fig. 1. Two diagnostic x-ray tubes are mounted to the room wall and ceiling orthogonal to one another, in the plane of gantry rotation. Each tube has a dot graticule which projects to the isocenter. The source to isocenter distances for the wall and ceiling tubes are about 3 and $2 \mathrm{~m}$, respectively. The reference images for patient alignment are digitally reconstructed radiographs (DRRs) obtained from the patient's treatment planning CT study. The DRRs are produced using extended source-axis distances so that the divergent geometry of each corresponding pair of images is consistent.

The imager itself is portable and consists of an orthogonal pair of phosphorescent screens (Kodak Lanex, Rochester, NY) taken from a standard film cassette (the front screen is used in the anterior-posterior view and the back screen is used in the lateral view for a supine patient), a pair of frontsurface mirrors, a charge-couple device (CCD) camera (SpectraSource Model MCD1200, Westlake Village, CA) with an $F / 1.2$ lens designed for $35 \mathrm{~mm}$ photography, and a sliding front-surface mirror that allows the camera to alternately receive images from one screen or the other. The CCD has a $1024 \times 1024$ array of 24 micron pixels with $75 \%$ quantum efficiency at $550 \mathrm{~nm}$ and a 12 bit dynamic range. It is thermoelectrically cooled to $-30{ }^{\circ} \mathrm{C}$. The field of view is 39 $\mathrm{cm}$ in diameter at the screen. Images are usually rebinned into $512 \times 512$, making the effective pixel size at the screen $0.8 \mathrm{~mm}$. The camera is controlled by a computer using a vendor-supplied software library and user-written software. The camera is shielded with $6 \mathrm{~mm}$ of lead to reduce direct detection of scattered x-rays by the CCD.

Room-based diagnostic imaging systems have been described for use in radiosurgery using a small X-band accelerator mounted on a robot $\operatorname{arm}^{14}$ and for head and neck treatments using implanted markers. ${ }^{15}$ The present system is intended to be flexible enough so that it can be used on a 




FIG. 1. Schematic drawing of the imaging system.

routine, day-to-day basis for treatments of any anatomic site, in a manner similar to alignment lasers. Since the alignment algorithm is based on curve matching of bony anatomy, this system does not require the use of markers, although they may be used for more precise localization. ${ }^{16}$

Since images are transported from the phosphor to the camera via a mirror-lens system, aberrations or misalignment of the optical components would introduce geometric distortions which could in principle lead to patient misalignment. It is therefore necessary to quantify the extent of geometric distortions in the imaging system. Using the full 1024 $\times 1024$ resolution of the CCD, images were taken of an array of spherical $2 \mathrm{~mm}$ diameter lead markers, spaced every $5 \mathrm{~cm}$ in each direction, placed directly in contact with the AP and lateral phosphor screens. As a simple visual check, the images were superimposed on a grid where regularly spaced markers were added in software. Quantitatively, the center of each marker was estimated interactively to the nearest pixel $(0.4 \mathrm{~mm})$, and difference vectors between the centers of nearest neighbor markers were measured. For both AP and lateral images, the spacing of the markers is constant to within $0.4 \mathrm{~mm}$ (one standard deviation) over the field of view. This is consistent with the uncertainty in measurement and placement of the markers.

The spatial resolution of the system, as measured with a high-contrast wire mesh phantom (Radiation Measurements Inc, Middleton, WI), is 1.25 line pairs $/ \mathrm{mm}$. At the extended source-screen distances used, the dependence of the spatial resolution on the focal spot size (measured to be about 1.0 $\mathrm{mm}$ ) is small. AP and lateral images were taken of anthropomorphic head, chest, and pelvis phantoms. The best-case (AP chest) and worst-case (lateral pelvis) images are shown in Fig. 2. Both images were processed with window and level adjustment only. There is some loss of contrast due to veiling glare in the lateral pelvis image. Care must be taken
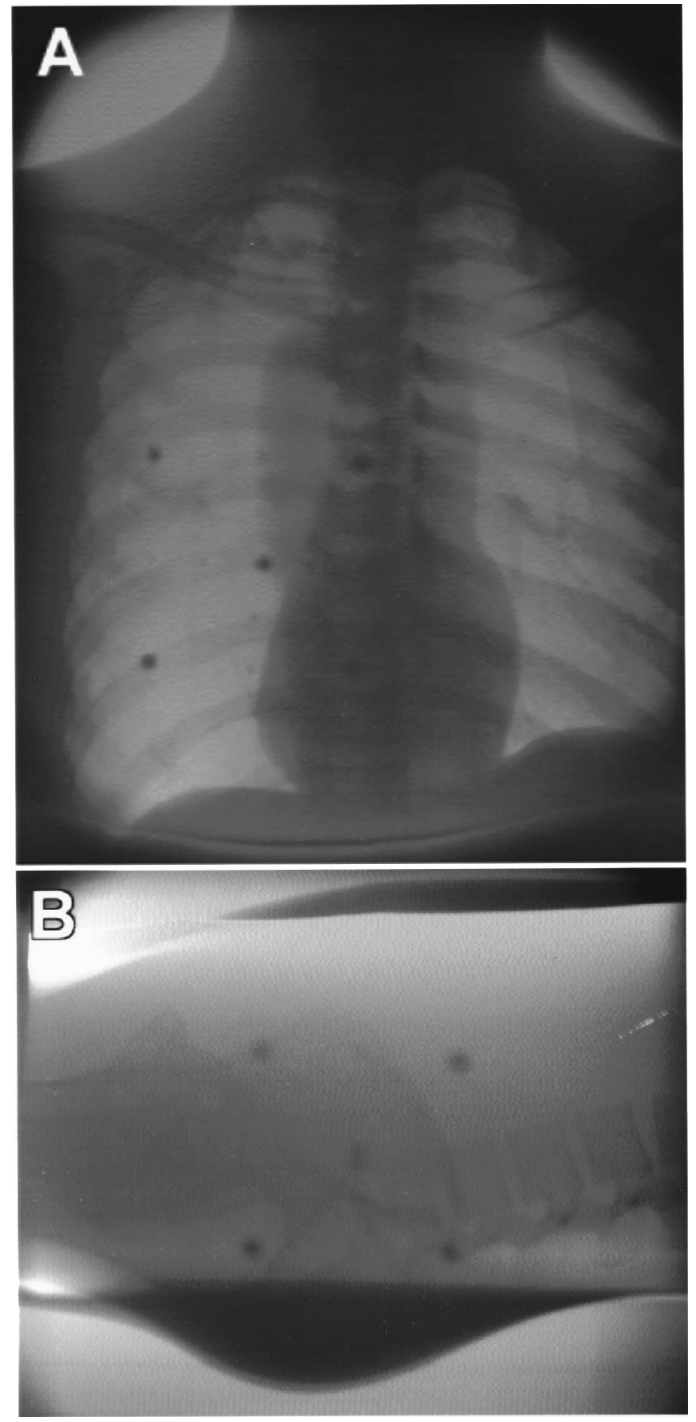

FIG. 2. (a) AP image of chest phantom, $220 \mathrm{mR}, 80 \mathrm{kVp}$, rebinned to 512 $\times 512$. (b) Lateral image of pelvis phantom, $1060 \mathrm{mR}, 120 \mathrm{kVp}$, rebinned to $512 \times 512$.

to minimize the area of the $\mathrm{x}$-ray field outside the patient to minimize this effect.

The imager is mobile and aligned visually using marks on the imager cart and on the floor. Since the coordinate system is established using dot graticules that are fixed to the wall and ceiling, measurements are expected to be insensitive to variations in the orientation of the cart. The sliding mirror moves only in its own plane, so measurements should also be insensitive to variations in mirror position. To verify these assumptions, two sets of images were taken of a stereotactic radiosurgery phantom rigidly mounted near the isocenter, using the full $1024 \times 1024$ resolution of the camera. The phantom position relative to the dot graticule system was measured using the algorithm described by Balter et al. ${ }^{11}$ First, seven orthogonal pairs of images were taken with the imager removed and then rolled roughly back into place between measurements. The variation in imager position was on the order of $2-3 \mathrm{~cm}$. Second, nine images were taken with the mirror moved in and out of place between measurements, at 
TABLE I. Results of alignment tests with stereotactic radiosurgery ball phantom. Quoted uncertainties are the standard deviation about the observed mean position. Translations are in $\mathrm{mm}$; rotations are in degrees.

\begin{tabular}{lcccc}
\hline \hline \multicolumn{1}{c}{ Test } & \# of alignments & $x$ translation & $y$ translation & rotation \\
\hline Self-alignment & 12 & 0.5 & 0.7 & 0.8 \\
Mirror & 8 & 0.5 & 1.0 & 1.0 \\
Cart (AP) & 6 & 0.7 & 0.5 & 0.9 \\
Cart (lateral) & 6 & 0.3 & 0.8 & 0.6 \\
\hline \hline
\end{tabular}

a fixed cart position. For each set, the image to which the others were aligned was chosen arbitrarily. To estimate the uncertainty in the alignment process, a single image was aligned to itself repeatedly. The results are shown in Table I. Within the uncertainty in alignment, there is little or no observed variation in image position with variations in cart position or repeated placement of the mirror.

In summary, random and systematic uncertainties in image position due to optical distortions and variations in the cart or mirror positions are less than $1 \mathrm{~mm}$, without precisely measuring the cart position or applying corrections for artifacts. As discussed earlier, the uncertainty in alignment of patient anatomy using the method of Ref. 11 is about 1.2 $\mathrm{mm}$. Patient setup variations are on the order of 5-7 $\mathrm{mm}$ at one standard deviation for each of three translations. ${ }^{1-6}$ Therefore the room coordinate system as defined by the dot graticules and measured by the imaging system is sufficiently accurate and precise to be used for routine measurement of patient setup.

The authors wish to thank David Hornick and Dale Litzenberg for assistance with the installation and alignment of the $\mathrm{x}$-ray tubes and dot graticule system. This work was supported by NIH Grant No. P01-CA59827. Presented in part at the annual meeting of the American Association of Physicists in Medicine, Boston, MA, 22-27 July 1995.

\footnotetext{
${ }^{\text {a)} E l e c t r o n i c ~ m a i l: ~ j s c h e w e @ u m i c h . e d u ~}$
}

${ }^{1}$ J. E. Schewe, J. M. Balter, K. L. Lam, and R. K. Ten Haken, "Measure- ment of patient setup errors using port films and a computer-aided graphical alignment tool,' Med. Dosim. 21, 97-104 (1996).

${ }^{2}$ J. E. Marks and A. G. Haus, "The effect of immobilisation on localisation error in the radiotherapy of head and neck cancer,' Clin. Radiol. 27, 175-177 (1976).

${ }^{3}$ I. Rabinowitz, J. Broomberg, M. Goitein, K. McCarthy, and J. Leong, "Accuracy of radiation field alignment in clinical practice," Int. J. Radiat. Oncol., Biol., Phys. 11, 1857-1867 (1985).

${ }^{4}$ H. Huizenga, P. C. Levendag, P. M. Z. R. De Porre, and A. G. Visser, "Accuracy in radiation field alignment in head and neck cancer: A prospective study," Radiother. Oncol. 11, 181-187 (1988).

${ }^{5}$ C. L. Creutzberg, V. G. M. Althof, H. Huizenga, A. G. Visser, and P. C. Levendag, "Quality assurance using portal imaging: The accuracy of patient positioning in irradiation of breast cancer," Int. J. Radiat. Oncol., Biol., Phys. 25, 529-539 (1993).

${ }^{6}$ A. A. H. El-Gayed, A. Bel, R. Vijlbrief, H. Bartelink, and J. V. Lebesque, "Time trend of patient setup deviations during pelvic irradiation using electronic portal imaging," Radiother. Oncol. 26, 162-171 (1993).

${ }^{7}$ J. M. Balter, G. T. Y. Chen, C. A. Pelizzari, S. Krishnasamy, S. Rubin, and S. Vijayakumar, "Online repositioning during treatment of the prostate: a study of potential limits and gains," Int. J. Radiat. Oncol., Biol., Phys. 27, 137-143 (1993).

${ }^{8}$ P. J. Biggs, M. Goitein, and M. D. Russell, "A diagnostic x-ray field verification device for a 10 MV linear accelerator,” Int. J. Radiat. Oncol., Biol., Phys. 11, 635-643 (1985).

${ }^{9}$ A. S. Shiu, K. R. Hogstrom, N. A. Janjan, R. S. Fields, and L. J. Peters, "Technique for verifying treatment fields using portal images with diagnostic quality,' Int. J. Radiat. Oncol., Biol., Phys. 18, 1589-1594 (1987).

${ }^{10}$ D. A. Jaffray, K. Chawla, C. Yu, and J. W. Wong, "Dual-beam imaging for online verification of radiotherapy field placement," Int. J. Radiat. Oncol., Biol., Phys. 33, 1273-1280 (1995).

${ }^{11}$ J. M. Balter, C. Pelizzari, and G. T. Y. Chen, "Correlation of planar images in radiotherapy using open curve segments and point pairs," Med. Phys. 19, 329-334 (1992).

${ }^{12}$ B. A. Fraass, D. L. McShan, M. L. Kessler, G. M. Matrone, J. D. Lewis, and T. A. Weaver, "'A computer-controlled conformal therapy system. I: Overview," Int. J. Radiat. Oncol., Biol., Phys. 33, 1139-1157 (1995).

${ }^{13}$ D. Hornick, D. W. Litzenberg, K. L. Lam, J. M. Balter, J. Hetrick, and R. K. Ten Haken, "A tilt and roll device for automated correction of rotational setup errors," Med. Phys. 25, 1739-1740 (1998).

${ }^{14}$ M. J. Murphy and R. S. Cox, "The accuracy of dose localization for an image-guided frameless radiosurgery system,"' Med. Phys. 23, 20432049 (1996)

${ }^{15}$ K. P. Gall, P. Zygmanski, and A. F. Thornton, “A system for diagnostic quality radiographic alignment of radiotherapy patients,' Int. J. Radiat. Oncol., Biol., Phys. 36, 206 (1996).

${ }^{16}$ K. L. Lam, R. K. Ten Haken, D. L. McShan, and A. F. Thornton, “Automated determination of patient setup errors in radiation therapy using spherical radio-opaque markers," Med. Phys. 20, 1145-1152 (1993). 